\title{
Single Step Test for Diagnosing Gestational Diabetes Mellitus
}

\author{
${ }^{1}$ Krishna Dahiya, ${ }^{2}$ Jyoti Sahu
}

\begin{abstract}
Gestational diabetes mellitus is one of the most common medical disorders found in pregnancy. Clinical recognition of GDM is important because timely intervention by dietary measures or insulin and fetal surveillance can reduce the well described associated maternal and fetal complications. This observational study was done in PGIMS, Rohtak during period from January 2011 to December 2011. Single step test using $75 \mathrm{gm}$ oral glucose was used as screening and diagnostic test for GDM. Fetomaternal outcome was studied in all the subjects. Prevalence of GDM was $7 \%$. In Indian context with a high prevalence of GDM the Universal screening is superior to selective screening in detecting more cases, facilitating early diagnosis and associated with improved pregnancy outcome with appropriate management. Present study concurs with the WHO recommendation of 2 hours $75 \mathrm{gm}$ OGTT as single step procedure for both screening as well as diagnosis of GDM. Hence, we suggest the adaptation of $75 \mathrm{gm}$ glucose single step test for screening and diagnosis of GDM.
\end{abstract}

Keywords: Gestational diabetes mellitus, Single step test, Universal screening, Fetomaternal outcome.

How to cite this article: Dahiya K, Sahu J. Single Step Test for Diagnosing Gestational Diabetes Mellitus. J South Asian Feder Obst Gynae 2014;6(2):88-92.

Source of support: Nil

Conflict of interest: None declared

\section{INTRODUCTION}

Gestational diabetes mellitus (GDM) is defined as carbohydrate intolerance of variable severity with onset or first recognition during pregnancy and usually resolves not long after delivery. ${ }^{1}$ GDM has serious long-term consequences for both baby and the mother, including a predisposition to obesity, metabolic syndrome, type-2 diabetes and cardiovascular diseases later in life. ${ }^{2}$ GDM offers an important opportunity for the development, testing and implementation of clinical strategies for diabetes prevention. Timely taken action in

${ }^{1}$ Professor, ${ }^{2}$ Resident

1,2Department of Obstetrics and Gynecology, Pandit Bhagwat Dayal Sharma Post Graduate Institute of Medical Sciences Rohtak, Haryana, India

Corresponding Author: Krishna Dahiya, Professor Department of Obstetrics and Gynecology, 74-R, Model Town Rohtak-124001, Haryana, India, Phone: 01262211888, e-mail: krishnadahiya@rediffmail.com, drkrishnadahiya@gmail.com screening of all pregnant women for glucose intolerance, achieving euglycemia in them and ensuring adequate nutrition may prevent the vicious cycle of transmitting glucose intolerance from one generation to another. The frequency of GDM usually reflects the frequency of type-2 diabetes in underlying population. ${ }^{3}$ GDM is one of the most common medical complications of pregnancy with an overall prevalence of 3 to $5 \%$ but as high as $18 \%$, depending on the population tested, screening methods employed and the diagnostic criteria used. ${ }^{4}$ However, an accurate estimation of global incidence of GDM in many countries does not exist because of lack of uniform standards of testing around the world, which could lead not only to under diagnosis but also under management of the illness. Controversy exists regarding optimal strategy for the detection and diagnosis of GDM. The American Diabetes Association (ADA) recommends two step procedures for screening and diagnosis of diabetes in selective population. Compared with selective screening, universal screening for GDM detects more cases and improves maternal and offspring prognosis. ${ }^{5}$ In Indian scenario, screening is essential in all pregnant women as Indians have 11-fold increased risk of developing glucose intolerance as compared to Caucasian. ${ }^{6}$ Seldom, a pregnant woman visiting the ante-natal clinic for the first time comes in the fasting state. It is suggested in few studies that the screening to diagnose GDM in first trimester and early care results in a better outcome. In Indian setting, one-step WHO procedure is suitable as the pickup rate is three times more than ADA criteria. This one step test is also better in terms of saving time, limiting cost on repeated visits to health center and reducing repeated invasive sampling. Hence, it is important to have a test that detects the glucose intolerance irrespective of fasting state and also has diagnostic accuracy. One Step Test called the DIPSI (Diabetes in Pregnancy Study Group, India) test is established by Dr Seshiah and groups ${ }^{7}$ in India and has been validated and published and included in Indian Guidelines for GDM (2009). The single step procedure serves both as screening and diagnostic test for GDM, is simple, economical and feasible. Diabetes in pregnancy and awareness project (DIPAP) performed a community based study, in a cohort of 12,506 pregnant women. Among them the overall prevalence of GDM was $13.9 \%$. Our study was planned to evaluate the prevalence of GDM 
in North India using $75 \mathrm{gm}$ oral glucose in a single step test procedure with a single glucose value, which serves both as a screening as well as diagnostic tool of GDM.

\section{MATERIALS AND METHODS}

The present prospective study was conducted in the Department of Obstetrics and Gynecology of Pandit Bhagwat Dayal Sharma Post Graduate Institute of Medical Sciences, Rohtak. A total of 500 pregnant women attending antenatal clinic for routine antenatal check-up were selected randomly for the study at less than 16 weeks period of gestation. These females were recruited for the study during the period of Jan 2011 and Dec 2011. All the selected women were given a $75 \mathrm{gm}$ anhydrous glucose powder dissolved in a glass of water, to be consumed over 5 minutes, irrespective to the time of last meal. A venous blood sample was collected at 2 hours for estimating plasma glucose by the glucose oxidase peroxidase (GOD-POD) method. The subjects were asked to avoid physical activity and smoking for 2 hours after intake of glucose. Gestational diabetes mellitus was diagnosed if 2 hours plasma glucose is $>140 \mathrm{mg} / \mathrm{dl}$. If the glucose level was $>200 \mathrm{mg} / \mathrm{dl}$, she was labeled as overt diabetes. In women who were found to have normal glucose level at first antenatal visit, the test was repeated at around 24 to 28 weeks period of gestation. The women with positive test were treated for control of blood sugar as per hospital protocol, i.e. medical nutritional therapy (MNT) for 2 weeks and if MNT failed to achieve control then insulin was initiated. The patients were followed up till delivery. The fetomaternal outcome was studied in terms of antenatal (pre-eclampsia, polyhydramnios, preterm labor, vaginal infections, intrauterine growth restriction, congenital malformation, intrauterine death), perinatal (shoulder dystocia, vaginal delivery, cesarean section), postnatal (postpartum hemorrhage) and neonatal (macrosomia, Apgar score, respiratory distress, hypoglycemia, hyperbilirubinemia, birth injuries, admission to nursery) complications. Pregnant women suffering from chronic renal disease, pancreatic disease, thyroid disorder or other endocrinal disease, known case of diabetes mellitus or patient on medications affecting glucose metabolism such as progesterone, corticosteroids, psychoactive agents, catecholamines and women with substance abuse such as opioids, cocaine, marijuana and benzodiazepines were excluded from study. Statistical analysis was performed with help of Epi Info ${ }^{\mathrm{TM}}$ 3.5.3. Epi Info is a trademark of the centers for disease control and prevention (CDC). Chisquare test was used to test the association of different study variables with the study groups. Z-test was used to test the significant difference between two proportions of the groups. $\mathrm{t}$-test was used to compare the means. Odds ratio (OR) with
$95 \%$ confidence interval $(\mathrm{CI})$ was calculated to measure the different risk factor. Significance level was set at 0.05 and confidence intervals were at $95 \%$ level. p-value $<0.05$ was considered statistically significant.

\section{RESULTS}

The mean age of the study population was $24.54 \pm 3.62$ years. In the study population of 500 subjects, 302 (60.4\%) of the women were aged less than 25 years and remaining 198 (39.6\%) belonged to risk factor group (age $\geq 25$ ). Out of 500 subjects, 35 (7\%) had blood glucose value $\geq 140 \mathrm{mg} / \mathrm{dl}$ and positive by the single step test. Prevalence of GDM in our study population was found to be $7 \%$. Out of 35 women, $4(11.4 \%)$ were positive during the first trimester and 31 $(88.6 \%)$ were positive in the second trimester when the test was repeated. The mean age of the controls (non GDM) was $23.82 \pm 3.31$ years with range 19 to 38 years and the median age was 23 years. The mean age of the cases was $27.00 \pm$ 3.14 years with range 21 to 33 years and the median age was 28 years. The mean age of the cases was significantly higher than the mean age of the controls ( $t-5.76 ; \mathrm{p}<0.001)$. Age distribution shows that there was significant association between age and diagnostic distribution of the patients $(\mathrm{p}<0.00001)$. In controls 294 (63.2\%) women belongs to the age group 19 to 24 years and 171 (36.8\%) belonged to risk group of age $\geq 25$ years. In case of GDM group $8(22.9 \%)$ women were in age group of 19 to 24 years, where $27(77.1 \%)$ women were in risk group. Obesity was a major risk factor for development of GDM. There were 7 (20\%) women of GDM group, whereas only $8(1.7 \%)$ women of controls were in the risk group of obesity. The risk of being GDM patient was 14.28 times [OR-14.28 $(4.83,42.21) ; \mathrm{p}=0.0001]$ for the obese women compared to nonobese. Other risk factors associated with GDM were family history of DM and history of macrosomic baby in previous pregnancy. The risk factors for GDM were present in 32 (91.4\%) women out of 35 cases of GDM while

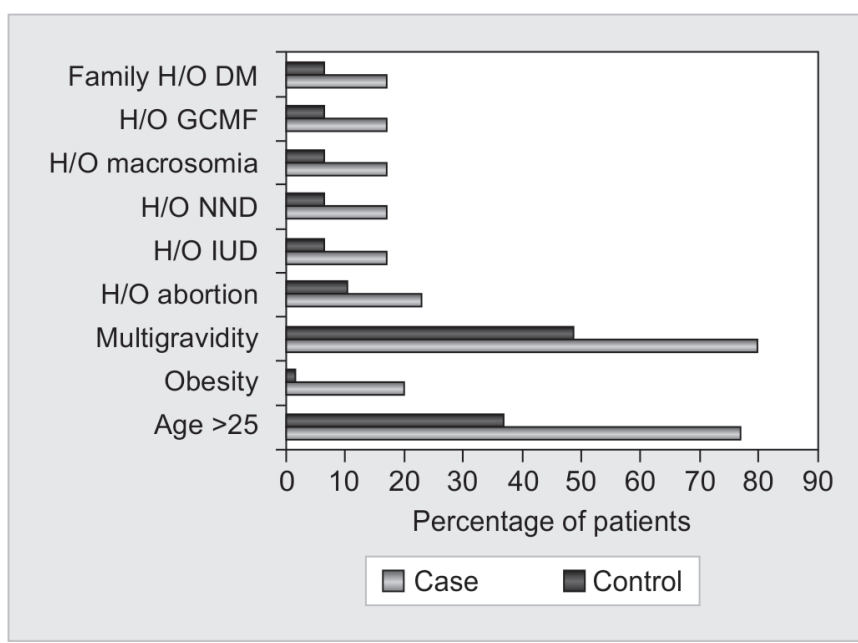

Graph 1: Prevalence of risk factors 
Table 1: Maternal complications in study population

\begin{tabular}{|c|c|c|c|c|c|}
\hline \multirow[t]{2}{*}{ Maternal outcome } & \multicolumn{2}{|c|}{ GDM group } & \multicolumn{2}{|c|}{ Control group } & \multirow[t]{2}{*}{ Odds ratio with $95 \% \mathrm{Cl}$} \\
\hline & No. & $\%$ & No. & $\%$ & \\
\hline Polyhydramnios & 6 & 17.1 & 7 & 1.8 & $11.20(3.53,35.51) ; p=0.0001$ \\
\hline Preterm labor & 3 & 8.6 & 21 & 5.4 & $1.62(0.46,5.75) ; p=0.44$ \\
\hline $\mathrm{PIH}$ & 5 & 14.3 & 31 & 8 & $1.91(0.69,5.26) ; p=0.20$ \\
\hline Vaginal infections & 4 & 11.4 & 10 & 2.6 & $4.85(1.43,16.36) ; p=0.005$ \\
\hline Oligohydramnios & 0 & 0 & 4 & 1 & NA \\
\hline $\mathrm{APH}$ & 0 & 0 & 2 & 0.5 & NA \\
\hline IUGR & 2 & 5.7 & 6 & 1.6 & $3.83(0.75,19.77) ; p=0.08$ \\
\hline GCMF & 1 & 2.9 & 6 & 1.6 & $1.86(0.21,15.92) ; p=0.56$ \\
\hline IUD & 2 & 5.7 & 16 & 4.1 & $1.40(0.21,5.61) ; p=0.66$ \\
\hline
\end{tabular}

Table 2: Neonatal outcome in both the study groups

\begin{tabular}{lllllll}
\hline \multirow{2}{*}{$\begin{array}{l}\text { Birth weight } \\
(\mathrm{kg})\end{array}$} & \multicolumn{2}{l}{ GDM group } & & \multicolumn{2}{l}{ Control group } & p-value \\
\cline { 2 - 3 } \cline { 5 - 6 } & No. & $\%$ & & No. & $\%$ & \\
\hline$<2.5$ & 5 & 14.29 & & 27 & 7 & $0.21, \mathrm{NS}$ \\
$2.5-3.99$ & 26 & 84.31 & & 352 & 92.2 & $0.001, \mathrm{~S}$ \\
$\geq 4$ & 4 & 11.4 & & 7 & 1.8 & $0.001, \mathrm{~S}$ \\
Total & 35 & 100 & & 386 & 100 & \\
Mean \pm SD & $3.154 \pm 0.282$ & & $2.850 \pm 0.395$ & $0.01, \mathrm{~S}$ \\
Apgar score & & & & & \\
At 1 minute & $6.12 \pm 0.98$ & & $6.82 \pm 0.65$ & $<0.001, \mathrm{~S}$ \\
At 5 minutes & $8.81 \pm 0.56$ & & $8.76 \pm 0.42$ & $>0.53, \mathrm{NS}$ \\
\hline
\end{tabular}

NS: Nonsignificant; S: Significant

$3(8.6 \%)$ of the cases did not have any of the risk factors (Graph 1). In control group, 289 (62.2\%) women were positive for risk factors and $176(37.8 \%)$ were without risk factors.

The fetomaternal outcome was studied in 35 cases and 386 controls. GDM positive females had overall poor outcome in the present pregnancy and significantly higher incidence of polyhydramnios and recurrent vaginal infections (Table 1). Cesarean section rate was significantly higher in the GDM cases (40\%) as compared to control group (15\%).

Neonatal outcome was poor in Babies born to GDM positive mothers, with higher incidence of macrosomia and lower apgar scoring at 1 minute (Table 2).

The fetal outcome was compared in both groups and was significantly poor in the GDM positive mothers. The incidence of macrosomia understandably was higher in GDM group. Metabolic derangements, like hypoglycemia, hyperbilirubinemia, were also higher in GDM positive mothers. Due to all these problems in the newborn, the admission to NICU were also proportionately higher in GDM positive females (Table 3).

\section{DISCUSSION}

Gestational diabetes mellitus is one of the most common medical disorders found in pregnancy. Clinical recognition of GDM is important because timely intervention by dietary measures or insulin and fetal surveillance can reduce the well described associated maternal and fetal complications. The frequency of GDM is highly variable and generally reflects the underlying pattern of diabetes mellitus in that particular population. There is no universal agreement on the screening strategy and diagnostic criteria for GDM. This study was undertaken to detect the glucose intolerance that occurs during pregnancy using single step test which serve both as a screening and a diagnostic tool at the same time and which is acceptable, economical and feasible to perform in the India. The two step test of screening with 50 gm glucose challenge test (GCT) and then diagnosing with oral glucose tolerance test (OGTT) is not feasible in our country, because the pregnant women may have to visit the antenatal clinic twice and multiple blood samples have to be withdrawn, which they resent and the 'no show' rate is high. ${ }^{8}$

The mean age of the pregnant women in the study population was $24.54 \pm 3.62$ years. In GDM group, the prevalence increased with age from $22.9 \%$ in the age group of 25 years to $77.1 \%$ in the age group $\geq 25$ years. So as the age increases the incidence of GDM increases. A number of investigators have found that maternal age is highly correlated with the risk of GDM. ${ }^{9,10}$ GDM was diagnosed in $12.4 \%$ women within 16 weeks of gestation, $23 \%$ between 17 and 23 weeks and remaining $64.6 \%$ at more than 24 weeks of gestation by Seshiah et al. ${ }^{11}$ In the present study $4(11.4 \%)$ women were diagnosed with GDM on their

Table 3: Neonatal morbidity in present pregnancy

\begin{tabular}{|c|c|c|c|c|c|}
\hline \multirow[t]{2}{*}{ Fetal outcome } & \multicolumn{2}{|c|}{ GDM group } & \multicolumn{2}{|c|}{ Control group } & \multirow[t]{2}{*}{ Odds ratio with $95 \% \mathrm{Cl}$} \\
\hline & No. & $\%$ & No. & $\%$ & \\
\hline Macrosomia & 4 & 11.4 & 7 & 1.8 & $6.98(1.93,25.17), p=0.0006$ \\
\hline RDS & 2 & 5.7 & 4 & 1 & $5.78(1.02,32.78), p=0.02$ \\
\hline Hyperbilirubinemia & 4 & 11.4 & 9 & 2.3 & $5.41(1.57,18.55), p=0.0002$ \\
\hline Hypoglycemia & 2 & 5.7 & 5 & 1.3 & $4.61(0.86,24.72), p=0.05$ \\
\hline Admission to NICU & 5 & 14.3 & 15 & 3.9 & $4.12(1.40,12.11), p=0.005$ \\
\hline
\end{tabular}


first visit, i.e. within 16 weeks of gestation and 31 (88.6\%) women on second visit, i.e. between 24 to 28 weeks of gestation. The prevalence of GDM ranges from 0.2 to $12 \%$ depending on the population studied. Seshiah et al screened 3674 pregnant women with 2 hours 75 gm test in various parts of the country and an overall prevalence of $16.55 \%$ was found. ${ }^{8}$ In a study by Zargar et al the prevalence of GDM in Kashmiri women was $3.8 \%{ }^{12}$ In present study prevalence of GDM was found to be $7 \%$. The prevalence of this study is comparable to Jindal et $\mathrm{al}^{9}$ and Das et al ${ }^{10}$ studies. In the present study $22(77.1 \%)$ of GDM positive women were of age $\geq 25$ years, while in the control group only $294(36.8 \%)$ were in this age group. Seshiah et al in their community based study found the highest prevalence in the age group of 30 to 34 years. ${ }^{11}$ Zargar et al also found that GDM prevalence increased steadily with increasing age (from $1.7 \%$ in women below 25 years to $18 \%$ in women 35 years or older). ${ }^{12}$ In this regard our study corresponds to findings of these authors. In our study seven (20\%) of cases were obese as compared to only $8(1.7 \%)$ of the control population. Various authors have confirmed that not only obesity but also overweight women have greatly increased risk of developing gestational diabetes. . $^{8,11,12}$

Out of 500 subjects, 421 females delivered in our institute. As expected, women with GDM in the present study were found to have higher proportion of obstetric complications including polyhydroamnios (11.2 times), recurrent vaginal infections (4.85 times), intrauterine growth retardation (3.86 times), intrauterine death (1.4 times) preterm labor (1.62 times), preeclampsia (1.91 times) and GCMF (1.86 times). Similar findings were found by Ganguly et al, Turki G and Odar et al in their respective studies. ${ }^{13-15}$ The effect of maternal hyperglycemia at conception or in period of embryogenesis usually manifests itself as congenital anomalies where as glucose intolerance during pregnancy leads to large for gestational age fetuses, metabolic disturbances, fetal demise and need for admission in NICU. Tahir et al found $28.7 \%$ rate of macrosomia in a study of GDM patients. ${ }^{16}$ The rate of large for gestational age babies in study by Akhlaghi and Hamedi was 14.3 and $16 \%$ in Ray et al study. ${ }^{17,18}$ In the present study, incidence of macrosomia was 4 (11.4\%) in GDM patients as compared to 7 (1.8\%) in control group. Metabolic complications like neonatal hypoglycemia, polycythemia, hypocalcemia and hyperbilirubinemia may complicate GDM babies. In our study GDM babies suffered more of these complications than the babies of the control group. Hypoglycemia was seen in 5.7\%, hyperbilirubinemia in $11.4 \%$, respiratory distress syndrome in $5.7 \%$ babies born to GDM mothers. In an Iranian study the incidence of respiratory distress syndrome, hypoglycemia and large for gestational age baby were $3.7,18.5$ and $14.8 \%$ respectively in the GDM population. ${ }^{17}$

Uncertainty exists regarding the value of diagnosis and the target population for GDM testing, i.e. whether universal screening should be done or it should be based on risk factors identified. Screening by risk factors alone has a sensitivity of $63 \%$ and a specificity of $56 \%$. In other words, 37 to $50 \%$ of women with GDM may go undiagnosed using this approach. In our study $8.6 \%$ of the GDM positive women did not have any of the risk factors which predispose to GDM and these cases could have been missed if selective screening had been done based on presence of risk factors. Though the argument can be raised that about $37 \%$ women in control group, who did not have any of the risk factors underwent the screening test unnecessarily as universal screening was followed in our study. One of the drawbacks of two step procedure is 'no show' for the second test. In a study by Seshiah et al $28.7 \%$ women did not return for OGTT after the previous GCT. ${ }^{19}$ Magee et al reported that in their follow-up 91 of the 457 positive screen individuals failed to undergo diagnostic test. ${ }^{20}$ de Aguiar et al also observed in their study that $23 \%$ of their screen positive women did not return for OGTT. ${ }^{21}$ Also the requirement of a number of blood samples for diagnosis of GDM is not feasible and conducive especially in the Indian context.

\section{CONCLUSION}

GDM is an important public health priority in the prevention of diabetes is to address maternal health both during antenatal and postpartum period. In Indian context with a high prevalence of GDM the universal screening is superior to selective screening in detecting more cases, facilitating early diagnosis and associated with improved pregnancy outcome with appropriate management. Present study concurs with the WHO recommendation of 2 hours 75 gm OGTT as single step procedure for both screening as well as diagnosis of GDM. Hence, we suggest the adaptation of 75 gm glucose single step test for screening and diagnosis of GDM. This test is done irrespective of fasting state and causes least disturbance in pregnant women's routine activities. This single step test is simple, economical and feasible and would be useful in effective management strategy for better maternal and fetal outcome.

\section{REFERENCES}

1. American Diabetes Association: Position statement - gestational diabetes. Diabetes Care 1986;9:430-431.

2. Reece EA, Leguizamon G, Wiznitzer A. Gestational diabetes: the need for a common ground. Lancet 2009;373:1789-1797.

3. Ferrara A. Increasing prevalence of gestational diabetes mellitusa public health perspective. Diabetes Care 2007;30:141-146. 
4. Meltzer SJ, Snyder J, Penrod JR, Nudi M, Morin L. Gestational diabetes mellitus screening and diagnosis: a prospective randomized controlled trial comparing costs of one-step and two-step methods. Br J Obstet Gynaecol 2010;117:407-415.

5. Cosson E. Screening and insulin sensitivity in gestational diabetes. Abstract volume of the 40th Annual Meeting of the EASD, September 2004;A 350.

6. Dornhorst A, Paterson CM, Nicholls JSD, Wadsworth J, Chiu D, Elkeles R, et al. High prevalence of gestational diabetes in women from ethnic minority groups. Diabet Med 1992;9:820-825.

7. Seshiah V, Sahay BK, Das AK, Shah S, Banerjee S, Rao PV, et al. Gestational diabetes mellitus - Indian guidelines. J Indian Med Assoc 2009;107:799-806.

8. Sheshiah V, Balaji V, Balaji MS. Gestational diabetes mellitus in India. JAPI 2004;52:707-711.

9. Jindal A, Ahmed F, Bhardwaj B, Chaturvedi B. Prevalence clinical profile and outcome of gestational diabetes mellitus. J Obst Gyn India 2001;51:46-49.

10. Das V, Kamra S, Mishra A, Agarwal A, Agarwal CG. Screening for gestational diabetes and maternal and fetal outcome. J Obstet Gynaec India 2004;54:449-451.

11. Seshiah V, Balaji V, Balaji MS, Paneerselvam A, Arthi T, Thamizharasi M, et al. Prevalence of gestational diabetes mellitus in South India (Tamil Nadu): a community based study. JAPI 2008;56:329-333.

12. Zargar AH, Sheikh MI, Bashir MI, Masoodi SR, Laway BA, Wani AI, et al. Prevalence of gestational diabetes mellitus in Kashmiri women from the Indian subcontinent. Diabetes Res Clin Pract 2004;66:139-145.
13. Ganguly A. A study of diabetes mellitus over 8 years. J Obstet Gynaecol India 1995;45:27-31.

14. Gasim T. Gestational diabetes mellitus: maternal and perinatal outcomes in 220 Saudi women. Oman Med J 2012;27: 140-144.

15. Odar E, Wandadwa J, Kiondo P. Maternal and fetal outcome of gestational diabetes mellitus in Mulago Hospital, Uganda. African Hlth Sci 2004;4:9-14.

16. Tahir S, Zafar S, Thontia S. Effect of various degrees of maternal hyperglycemia on fetal outcome. J Surg Pak Int 2011;16: 61-66.

17. Akhlagi F, Hamedi AB. Comparison of maternal and fetal/ neonatal complications in gestational and pre-gestational diabetes mellitus. Acta Medica Iranica 2005;43:263-267.

18. Ray JG, Vermeulen MJ, Shapiro JL, Kenshole AB. Maternal and neonatal outcomes in pregestational and gestational diabetes mellitus and the influence of maternal obesity and weight gain: the DEPOSIT study. Diabetes Endocrine Pregnancy Outcome Study in Toronto. QJM 2001;94:347-356.

19. Seshiah V, Balaji V, Balaji MS, Sekar A, Sanjeevi CB, Green A. One step procedure for screening and diagnosis of gestational diabetes mellitus. J Obstet Gynecol India 2005;55:525-529.

20. Magee S, Walden CE, Bendedetti TJ. Influence of diagnostic criteria on the incidence of gestational diabetes and perinatal morbidity. JAMA 1993;269:609-615.

21. de Aguiar LG, de Matos HJ, de Brito Gomes M. Could fasting plasma glucose be used for screening high-risk outpatients for gestational diabetes mellitus ? Diabetes Care 2001;24: 954-955. 